\title{
Use of Quinoline Alkaloids as Markers of the Floral Origin of Chestnut
}

Honey

PILAR TRUCHADO†; ISABEL MARTOS†; LAURA BORTOLOTTI\$; ANNA G.SABATINI + ;FEDERICO FERRERES $†$ AND FRANCISCO A. TOMAS-

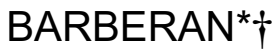

Research Group on Quality, Safety and Bioactivity of Plant Food, CEBAS-CSIC. P.O. Box 164, Espinardo (Murcia) 30100, Spain, and Consiglio per la Ricerca e la sperimentazione in Agricoltura, Unitá di Ricerca di Apicoltura e Bachicoltura, CRA-API, Via di Saliceto, 80, 40128 Bologna, BO, Italy.

*Corresponding author: Tel: +34-968-396334; Fax: +34-968-396213. e-mail: fatomas@cebas.csic.es

$\dagger$ †EBAS-CSIC.

†CRA-API. 


\section{Abstract}

In order to identify potential floral markers of chestnut honey, the phytochemicals present in chestnut floral nectar collected by bees were analyzed. Two nitrogen-containing compounds were detected, isolated and identified as 4-hydroxyquinaldic acid (kynurenic acid), and 4-quinolone-2carboxylic acid by ${ }^{1} \mathrm{H}$ NMR and ${ }^{13} \mathrm{C}$ NMR. In addition, chestnut nectar contained the monoterpene 4-(1-hydroxy-1-methyl-ethyl) cyclohexa-1,3-diene-1-carboxylic acid, and its gentiobioside ester and the flavonol quercetin 3-pentosyl-hexoside. These nectar markers were found in different chestnut unifloral honey samples, although the flavonol was not detected in all the samples analysed. The terpenoid derivatives had previously been found in linden and tilia honeys. These results show that quinoline alkaloids, and particularly 4-hydroxyquinaldic acid, are potentially good markers of chestnut honey, as they were not detected in any other unifloral honey analyzed so far. They are present at concentrations ranging from 6639 to $14638 \mu \mathrm{g} / 100 \mathrm{~g}$ honey. In addition, the terpenoid and flavonoid derivatives present in nectar, although not exclusively characteristic of this floral origin, are good complementary markers for the determination of the floral origin of chestnut honey.

Keywords: floral markers, botanical origin, nectar, chestnut, kynurenic acid, HPLC-MS-MS. 


\section{INTRODUCTION}

HPLC analysis of floral nectar phytochemicals is potentially an effective and objective method to help in honey floral origin determinations. This technique can complement the well established pollen and sensory analyses. Different studies have explored the application of the analysis of honey phytochemical constituents for this purpose. Thus, flavonoids and other phenolics $(1,2,3,4)$, volatile compounds (5), aromatic and degraded carotenoid-like substances $(6,7)$, aminoacids (8), aromatic aldehydes and heterocycles (9) have been evaluated to establish the floral origin of honey. The following phytochemicals have been proposed for the determination of honey floral origins: kaempferol for rosemary honey (10); myricetin, tricetin and luteolin for Eucalyptus honey (3); abscisic acid for heather honey (11); homogentisic acid for strawberry tree (Arbutus unedo) honey (12); methyl syringate for manuka honey (13); hydroxy quinaldic acid for chestnut honey (14); carboxylicmonoterpene glycosidic esters for Linden honey (15); hesperetin for Citrus honey (16); and kaempferol rhamnosides for Acacia (Robinia) honey (4).

Chestnut blossom honey production is important in many European countries. Verification of its floral origin is based essentially on sensory analysis, since there are no specific chemico-physical parameters for this kind of honey and the palynological analysis often inconclusive, since chestnut pollen is hyper-represented. Therefore, the identification of floral markers for chestnut honey is of great practical interest.

Chestnut honeys are dark in color and have a strong and characteristic flavour. Previous studies have suggested that volatile compounds, such as 3aminoacetophenone and 1-phenyl-ethanol, could be used as markers of the 
floral origin of chestnut honey (17). The occurrence of potential markers of chestnut honey was reported in a study of European unifloral honeys in which five unidentified markers were detected $(\mathrm{CH} 1-\mathrm{CH} 5)$ and showed characteristic UV spectra (18). These markers were not detected in any of the other unifloral honeys analyzed. One of these compounds was later identified as kynurenic acid $(14,19)$. However, in these previous studies, the occurrence of these markers, or their precursors, in chestnut nectar had not been explored due to the small size of chestnut flowers, and therefore the status of the floral origin markers was not confirmed.

The aim of the present study was the analysis of chestnut flower nectar phytochemicals by HPLC-DAD-MS-MS, using nectar collected by bees, and the evaluation of the possibility of using these compounds as markers of the botanical origin of chestnut honey.

\section{MATERIAL AND METHODS}

Reagents. Chlorogenic acid (5-O-caffeoylquinic acid), quercetin, and hesperetin were purchased from Sigma (St. Louis MO), and chrysin (5,7dihydroxyflavone) was from Carl Roth OGH (Karlsruhe, Germany). Formic and acetic acids were analytical grade and methanol was HPLC grade and supplied by Merck (Darmstadt, Germany). Milli-Q system (Millipore Corp., Bedford, MA) ultra pure water was used throughout this study.

Collection of Chestnut (Castanea sativa) nectar. Due to the small size of chestnut flowers, and to the relatively small amount of nectar that they produced, the collection of nectar directly from the flowers was not possible. Chestnut nectar was collected by placing the hives in a chestnut wood during 
the blooming period. After having checked from the combs that bees were collecting chestnut nectar, bees returning to the hive were captured and dissected, and their honey stomach was extracted and immediately frozen. About $1 \mathrm{~mL}$ of nectar was collected from nearly 50 honey bees.

Honey Samples. Seven chestnut honey samples from different Regions in Italy were analysed. These samples were provided and certified by the Agricultural Research Council - Bee and Silkworm Research Unit (CRA-API, Bologna, Italy). Origin of samples was mainly middle-north of Italy: three samples from Emilia Romagna (C-00, C-1390, C-1473); three samples from Tuscany, one from Siena (C-1453) and two from Arezzo (C-1454, C-1456); one sample from Piedmont (C-1757). All honey samples included in this study were stored in the dark at $4{ }^{\circ} \mathrm{C}$ until analysis.

Chestnut honey samples from France were used for isolation of the markers $\mathrm{CH} 1$ and $\mathrm{CH} 2$. This honey was from Bernard Michuaud S.A (Lune de miele) (Gan, France), and the pollen analysis revealed that the percentage of Castanea sativa pollen was higher than $90 \%$ in all cases.

Extraction of Markers from Nectar. Chestnut nectar collected from the bee stomach, was diluted with ultra pure water, and centrifuged at $7000 \mathrm{rpm}$ for $10 \mathrm{~min}$, in a Centromix centrifuge (Selecta, Barcelona). The supernatant was filtered through a reversed phase Sep-Pak solid phase extraction cartridge (a reversed phase $\mathrm{C}_{18}$ cartridge; Waters Milipore, USA). This cartridge was previously activated with $10 \mathrm{~mL}$ of methanol and then with $10 \mathrm{~mL}$ of water. The supernatant was filtered through the cartridge and washed with $10 \mathrm{~mL}$ water. The phytochemical compounds absorbed in the cartridge were then eluted with $1 \mathrm{~mL}$ methanol. The methanol fraction was filtered through a $0.45 \mu \mathrm{m}$ 
membrane filter Millex- $\mathrm{HV}_{13}$ (Millipore Corp., USA) and stored at $-20^{\circ} \mathrm{C}$ until further analysis by HPLC-DAD-MS-MS.

Extraction of phytochemical from Honey. Honey samples (10 g each) were mixed with five parts of water acidified to $\mathrm{pH}=2$ with $\mathrm{HCl}$ until completely fluid and filtered through cotton to remove solid particles. Then the filtrate was passed through a $\mathrm{C}_{18}$ cartridge (Sep-Pak) previously activated with methanol (10 $\mathrm{mL})$ and water $(10 \mathrm{~mL})$. The phenolic compounds and markers remained in the cartridge, while sugars and other polar compounds were eluted with the aqueous solvent. The cartridge was washed with distilled water $(10 \mathrm{~mL})$ and the phenolic fraction was then eluted with methanol $(2 \mathrm{~mL})$. The methanol extracts were stored at $-20^{\circ} \mathrm{C}$ until further analysis by HPLC-DAD-MS-MS.

HPLC-DAD-Tandem Mass Spectrometry (MS-MS) Analysis of Nectar and Honey Metabolites. All HPLC-MS analyses were achieved using an Agilent 1100 Series diode array and a mass detector in series (Agilent Technologies, Waldbronn, Germany). The HPLC system consisted of a binary pump (G1312 A), an auto sampler (G1313 A) a degasser (G1322 A), and photodiode-array detector (G1315 B) controlled by software (v. A08.03). The mass detector was an ion trap spectrometer (G2445A) equipped with an electrospray ionization (ESI) system and controlled by software (v. 4.1). The nebulizer gas was nitrogen, the pressure and the flow rate of the dryer gas were set at 65 psi and $11 \mathrm{~L} / \mathrm{min}$ respectively. The full scan mass covered the range from $\mathrm{m} / \mathrm{z}$ 100-1000. Collision-induced fragmentation experiments were performed in the ion trap using helium as collision gas, with voltage ramping cycles from 0.3 up to $2 \mathrm{~V}$. The capillary and voltage were maintained at $350^{\circ} \mathrm{C}$ and $4 \mathrm{kV}$, respectively. Mass spectrometry data were acquired in the negative 
mode for the identification of phenolic compounds and in the positive mode for the identification of nitrogen-containing markers of chestnut honey. The column used was a $250 \mathrm{~mm} \times 4 \mathrm{~mm}$ i.d., $5 \mu \mathrm{m}$, $\mathrm{C}_{18}$ LiChroCART RP-18 column protected with a $4 \mathrm{~mm} \times 4 \mathrm{~mm}$ i.d. LiChroCART guard column (Merck, Darmstadt, Germany). Elution was with water/formic acid (19:1 v/v) (solvent A) and methanol (solvent B). The elution was accomplished with a solvent flow rate of $1 \mathrm{~mL} / \mathrm{min}$, starting with $10 \% \mathrm{~B}$ to reach $30 \% \mathrm{~B}$ at $20 \mathrm{~min}, 45 \% \mathrm{~B}$ at $30 \mathrm{~min}, 60 \%$ $\mathrm{B}$ at $40 \mathrm{~min}, 70 \% \mathrm{~B}$ at $45 \mathrm{~min}, 90 \% \mathrm{~B}$ at $60 \mathrm{~min}$ and then became isocratic for 5 min. Chromatograms were recorded at 290, 320 and $340 \mathrm{~nm}$.

The potential markers of chestnut honey and flavonoids from propolis were identified according to their UV spectra, molecular weights, retention times and their MS-MS fragments, and wherever possible, chromatographic comparisons with authentic markers (commercial or previouly isolated and identified from honey or propolis). The markers $(\mathrm{CH} 1-\mathrm{CH} 3 ; \mathrm{CH} 5)$ were quantified as compound $\mathrm{CH} 1$ at $290 \mathrm{~nm}$. The flavanones were quantified as hesperetin at $290 \mathrm{~nm}$, the flavonols as quercetin and the flavones as chrysin at $340 \mathrm{~nm}$.

Isolation of Compounds $\mathrm{CH} 1, \mathrm{CH} 2$ and L1. To isolate and purify the chestnut honey markers $12 \mathrm{Kg}$ of chestnut honey from France were used. The extraction of the markers was achieved in $1 \mathrm{~kg}$ batches. $1 \mathrm{Kg}$ of honey was dissolved in water acidified to $\mathrm{pH} 2$ with $\mathrm{HCl}$ and mixed with $500 \mathrm{~g}$ of Amberlite XAD-2 (Supelco, Bellefonte; mean pore diam. 90 A, mesh size 20-60 $\mu \mathrm{m}$, mean surface area $300 \mathrm{~m}^{2} / \mathrm{g}$ ) up to ca. $3 \mathrm{~L}$ and stirred with a magnetic stirrer for 30 min to allow the adsorption of phenolic compounds. Then it was packed in a glass column $(84 \times 4 \mathrm{~cm})$ and was washed with acid water (c.a. $2 \mathrm{~L})$ to remove 
sugars and polar compounds and retain flavonoids and other honey phenolics. The column was washed with distilled water (c.a. 1 L). Phenolic compounds were then eluted with $\mathrm{MeOH}$ (c.a. $0.75 \mathrm{~L}$ ) until reaching a colourless eluent. The remaining water was submitted to additional adsorption on Amberlite XAD-2 to complete the recovery of phytochemicals from honey.

The whole sample $(12 \mathrm{Kg})$ was processed in this way. The methanol extracts from each extraction were combined and concentrated under reduced pressure down to $20 \mathrm{~mL}$ methanol. This extract was then chromatographed on a Sephadex LH-20 column (40 x $3 \mathrm{~cm})$ (Pharmacia, Upsala, Sweden) using methanol. The separation of different fractions was monitored using a $360 \mathrm{~nm}$ light, and the fractions obtained were analyzed by HPLC under the specific conditions for honey metabolites described above. The fractions containing the markers were joined and $\mathrm{CH} 1, \mathrm{CH} 2$ and $\mathrm{L} 1$ were isolated and purified by semipreparative HPLC on a Spherisorb octadecylsilane (ODS-2) column (25 x 1 $\mathrm{cm}, 5 \mu \mathrm{m}$ particle size) with a solvent flow rate of $5 \mathrm{~mL} / \mathrm{min}$ using as mobile phase methanol/water solutions in an isocratic manner starting with $10 \%$ methanol and increasing the methanol proportion to elute compounds (30\%). The purity of isolated compounds was tested by analytical HPLC and stored at room temperature after freeze-drying.

Phytochemicals Identification. The isolated unknown compounds were identified by a combination of UV spectrophotometry in methanol and, after the addition of the classical shift reagents (20), and by EIMS (Hewtlet packard electron impact mass spectrometer, $70 \mathrm{eV}$, direct inlet), ${ }^{1} \mathrm{H}$ NMR and ${ }^{13} \mathrm{C}$ NMR in DMSO- d6 (Brüker, $350 \mathrm{mHz}$ ). 


\section{RESULTS AND DISCUSSION}

Analysis of Compounds from Castanea sativa Nectar. The chestnut nectar obtained from the honey stomach of bees was analyzed by HPLC using a reverse phase $\mathrm{C}_{18}$ column, and its chromatographic profile is shown in Figure 1A. A total of six different compounds were identified as the main constituents of chestnut nectar. The chromatographic peaks, $\mathrm{CH} 1, \mathrm{CH} 2, \mathrm{CH} 5, \mathrm{~L} 1$ and $\mathrm{L} 2$ showed UV spectra with characteristic maxima and shoulders (Figure 1). These potential markers were previously detected in chestnut honey by our group in 2001 but they were not identified (18). In addition, compound $\mathrm{F}$ showed the characteristic UV spectrum of a flavonol.

The structures of $\mathrm{CH} 1, \mathrm{CH} 2$ and $\mathrm{L} 1$ were studied by means of a combination of their UV and mass spectra registered by HPLC-DAD-MS/MS ESI in positive and negative modes. The MS study of compound $\mathrm{CH} 1$ showed an ion at $\mathrm{m} / \mathrm{z} 190[\mathrm{M}+\mathrm{H}]^{+}$and its $\mathrm{MS} / \mathrm{MS}$ analysis showed that the most abundant fragment ion was that at $\mathrm{m} / \mathrm{z} 162$ that indicated the loss of $\mathrm{C}=\mathrm{O}$ (Figure 2A).The MS analysis of $\mathrm{CH} 2$ showed a molecular weight identical to that of $\mathrm{CH} 1\left(\mathrm{~m} / \mathrm{z} 190[\mathrm{M}+\mathrm{H}]^{+}\right)$. MS2 of this compound produced as the main fragment an ion at $m / z 172$ that indicated the loss of one molecule of $\mathrm{H}_{2} \mathrm{O}$ (Figure 2B). Its MS study, in the negative ion mode, showed that the pseudomolecular ion was at $m / z 188$ for $\mathrm{CH} 1$ and $\mathrm{CH} 2$. Their MS2 showed for both compounds that the most abundant ion was that corresponding to the loss of $\mathrm{HCOOH}(\mathrm{m} / \mathrm{z} 144)$, confirming the presence of a carboxyl residue, very probably bound to a substituted indole or hydroxylated (iso)quinaldic acid in both structures (Figure 3). The fragmentation of $\mathrm{CH} 1$ (Figure 2A) is in agreement with the results reported by other authors which indicated that this 
compound could be 4-hydroxyquinaldic acid or kynurenic acid $(14,21)$. However, the molecular structure of $\mathrm{CH} 2$ could not be identified by its $\mathrm{MS}$ studies. To confirm the definitive structures it was necessary to isolate and analyze these markers by ${ }^{1} \mathrm{H}$ NMR and ${ }^{13} \mathrm{C}$ NMR. Thus compounds $\mathrm{CH} 1, \mathrm{CH} 2$ and L1 were isolated by preparative column chromatography.

Compound $\mathrm{CH} 1$ showed a UV spectrum 340sh, 335sh, 332, 308, 282sh (Figure 1) consistent with that recorded with HPLC-DAD. Its EIMS showed a molecular ion at $\mathrm{m} / \mathrm{z} 189$ confirming that this was a nitrogen-containing compound. Its MS spectrum is shown in Table 1 and a MS spectra library search showed that this compound could be tentatively identified as 4-hydroxy2-quinolinecarboxylic acid (4-hydroxy quinaldic acid). The ${ }^{1} \mathrm{H}$ NMR analysis shows the presence of five aromatic protons in the molecule. The chemical shifts and coupling constants were in agreement with those reported for kynurenic acid (19) (Table 1) supporting the structure suggested above. The ${ }^{13} \mathrm{C}$ NMR spectrum showed the response of a carboxylic carbon (163.8 ppm), and the rest of the assignments were consistent with previously published data for this compound (Table 1) (19).

The isolated compound $\mathrm{CH} 2$ showed a UV spectrum 330sh, 309, 283 sh. This spectrum was similar to that of $\mathrm{CH} 1$, but the maxima had shorter wavelengths (Figure 1) indicating a molecule with less resonance. Its MS spectrum showed a molecular ion at $m / z 189$, showing that this was an isomer of compound $\mathrm{CH} 1$. Its ${ }^{1} \mathrm{HNMR}$ spectrum showed five protons as that of compound $\mathrm{CH} 1$ but the chemical shifts were quite different (Table 1). This was particularly relevant for the hydrogens at $\mathrm{H}-3$ and $\mathrm{H}-6$. The ${ }^{13} \mathrm{C}$ NMR spectrum was very close to that of $\mathrm{CH} 1$, suggesting a very similar molecule. The 
differences were observed for the carbons at $\mathrm{C}-2$ and $\mathrm{C}-10$. As this is a nitrogen-containing compound the possibility of protonation of the nitrogen to give a quinaldinium cation exists (Figure 4). This can produce the quinolone tautomer that could explain the differences observed in the UV spectrum and the differences in the NMR data. The presence of a proton in a nitrogen atom affects considerably the electronic environment of the protons in the quinaldic acid molecule. The interconversion of compounds $\mathrm{CH} 2$ and $\mathrm{CH} 1$ in acid solution supports the hypothesis that $\mathrm{CH} 2$ is the tautomer form of compound $\mathrm{CH} 1$ (Figure 4). Its ${ }^{13} \mathrm{C}$ NMR spectrum (Table 1) is consistent with a compound with this molecular structure.

Compound $\mathrm{CH} 5$ was detected as a minor peak in the UV chromatogram at $290 \mathrm{~nm}$ (Figure 1).The HPLC-MS study in positive mode showed that its [M-H] ion was at $m / z 241$. The MS2 analysis of this ion showed a fragment at $m / z 213$ corresponding to the loss of $\mathrm{C}=\mathrm{O}$. $\mathrm{CH} 5$ could not be identified due to the small amount present that prevented its isolation for ${ }^{1} \mathrm{H}$ NMR and ${ }^{13} \mathrm{C}$ NMR analyses. The MS study, UV spectra and HPLC retention time suggest that this compound could be an intermediate of the tryptophan metabolism pathway, similar to $\mathrm{CH} 1$ and $\mathrm{CH} 2$.

The UV spectrum of compounds $L 1$ and $L 2$ with a maximum at $305 \mathrm{~nm}$, suggests that these could coincide with the markers of tilia and linden unifloral honeys which have been previously described in other studies $(15,18)$. The first one was the most abundant compound detected in nectar and the second one was detected in smaller proportions (Figure 1). The MS analysis of L1 showed a deprotonated ion at $\mathrm{m} / \mathrm{z} 505$. The MS2 scan of the $[\mathrm{M}-\mathrm{H}]]^{-}$ion yielded two fragment ions at $m / z 323$ and $m / z$ 181. The first fragment indicated the loss of 
the terpenoid aglycone $(\mathrm{m} / \mathrm{z} 181)$ and the second one was obtained as result of the loss of two sugar moieties $(\mathrm{m} / \mathrm{z} 181)$. This fragmentation confirmed that $\mathrm{L} 1$ was the 1-O- $\beta$-gentiobioside ester of the monoterpene. The NMR analysis 4-(1hydroxy-1-methyl-ethyl) cyclohexa-1,3-diene-1-carboxylic (15) (Figure 3). The MS study of L2 showed that this had an ion at $\mathrm{m} / \mathrm{z} 181$ but its ionization was poor under the analytical conditions used. MS2 analysis showed a main fragment at $m / z 163$, due to the loss of water, $m / z 152$ due to the loss $C=0$, and $m / z 135$ due to the loss of $\mathrm{HCOOH}$. The molecular ion and fragments supported that compound L2 was the monoterpene 4-(1-hydroxy-1-methyl-ethyl) cyclohexa-1,3-diene-1-carboxylic acid, and this was in agreement with other authors $(15,22)$. $L 2$ was released after saponification of $L 1$.

Compound $F$ was detected in trace amounts (Figure 1). The MS analysis showed a deprotonated molecular ion at $\mathrm{m} / \mathrm{z} 595$ consistent with a quercetin pentosyl-hexoside. Both sugars are linked to one phenolic hydroxyl as the disaccharide moiety is released in one single fragment. The UV spectra indicated that the hydroxyl in 3 position is blocked (20).

Quantification of markers in chestnut honey. The HPLC analyses of chestnut honey extracts showed that the different compounds $(\mathrm{CH} 1, \mathrm{CH} 2, \mathrm{CH} 5$ L1 and L2) present in chestnut nectar were the main peaks in the chromatogram of chestnut honey samples (Figure 1B) and were characterized by HPLC-MS-MS. All these compounds were previously reported, although not identified), in a study of European honey where potential floral origin markers were detected ( $\mathrm{CH} 1-\mathrm{CH} 5)(18)$. However, in the present study compound $\mathrm{CH} 4$ was not detected, and $\mathrm{CH} 3$ was only present as a minor constituent in honey. Both compounds $\mathrm{CH} 3$ and $\mathrm{CH} 4$ were not observed in nectar. Compounds $\mathrm{CH} 1$, 
$\mathrm{CH} 2$ and $\mathrm{CH} 5$ were detected in all honeys (Table 2). For $\mathrm{CH} 1$ the concentration in the samples analysed was about $58.0 \mathrm{mg} / 100 \mathrm{~g}$ of honey, for $\mathrm{CH} 24.8$ $\mathrm{mg} / 100 \mathrm{~g}$ of honey and for $\mathrm{CH} 52.0 \mathrm{mg} / 100 \mathrm{~g}$. In the nectar chromatogram (Figure 1)

In the nectar chromatogram (Figure 1) compound $\mathrm{CH} 2$ was observed as a minor constituent while in the honey chromatogram this marker was the most abundant (Figure 2). This could be explained by the relatively easy interconversion of both tautomers.

Compounds L1 and L2 were detected in chestnut nectar and in all chestnut honey samples analysed except C-1454. L1 was the main constituent in chestnut nectar but it was only observed in minor proportions in honey. In a previous study, these compounds were isolated from linden nectar and honey and were suggested as markers for this floral origin (15). Moreover, these compounds L1-L2 have been detected in studies on chestnut honey floral markers $(18 ; 22)$.

The flavonol pentosyl-hesoxide $F$, that was a derivative of quercetin analyzed in chestnut nectar, was only detected in some honey samples (C1473; C-1390; C-00) but in trace amounts.

The content of markers in the different chestnut honey samples was rather variable as during honey elaboration/ripening, these compounds could be degraded or transformed. Climatic conditions and geographical origin could modify the concentration of these markers in honey.

Propolis-derived flavonoid quantification in chestnut honeys. The propolis-derived flavonoid aglycones were also extracted and analyzed by HPLC on reverse-phase columns. All samples showed a similar and 
characteristic HPLC profile. The content of propolis-derived compounds was in general very small in all samples $(0.1-1.0 \mathrm{mg} / 100 \mathrm{~g})$, as it is shown in Table 3 . These results are in accordance with those previously reported for chestnut honey (18). The content of these compounds in the different chestnut honey samples is quite variable because it depends on the degree of propolis contamination in the hive and beeswax $(18,23)$.

In conclusion, these results show that nitrogen-containing phytochemicals, such as 4-hydroxy quinaldic acid, and the terpenoid and flavonoid derivatives detected in chestnut floral nectar collected by bees are detected in chestnut honey as well. These compounds have been identified and quantified in different amounts in all the honey samples analyzed and they can be suggested as suitable markers to be used for the determination of the floral origin of chestnut honey.

These results confirm that the identification of floral markers from nectar can be a useful tool to avoid fraud on honey authenticity as they help determine the botanical origin of honey, representing an added value to honey quality.

\section{ACKNOWLEDGEMENTS}

The authors are grateful to Dr J. L. Rios and D. Cortes, for their useful discussions regarding alkaloids identification.

\section{LITERATURE CITED}

(1) Amiot M.J.; Aubert, S.; Gonnet, M.; Tacchini, M. Honey phenolic compounds a preliminary study on their identification and quantification by families. Apidologie. 1989, 20, 115-125 
(2) Ferreres, F.; Tomas-Barberan, F.A.; Gil, M.I.; Tomas-Lorente, F. An HPLC technique for flavonoid analysis in honey. J. Sci. Food Agric. 1991, 56, 4956.

(3) Martos, I.; Ferreres, F.; Tomas-Barberan F.A. Identification of flavonoids markers for the botanical origin of Eucalyptus honey. J. Agric. Food Chem. 2000, 48, 1498-1502.

(4) Truchado, P.; Ferreres, F.; Bortolotti, L.; Sabbatini, A. G.; Tomás-Barberán, F. A. Nectar flavonol rhamnosides are floral markers of acacia (Robinia pseudacacia) honey. J. Agric. Food Chem. 2008. 56, 8815-8824.

(5) Radovic, B. S.; Careri, M.; Mangia, A.; Musci, M.; Gerboles, M.; Anklam, E. Contribution of dynamic headspace GC-MS analysis of aroma compounds to authenticity testing of honey. Food Chem. 2001, 72, 511-520.

(6) Tan, S.; Wilkins, A. L.; P.T.; McGhie, T.K. Extractives from New Zealand Unifloral honeys 2. Degraded carotenoids and other substances from heather honey. J. Agric. Food Chem. 1989, 37, 1217-1222.

(7) Wilkins, A.L.; Lu, Y.; Tan, S.T. Extractives from New Zealand honeys. 4. Linalool derivates and other components from nodding thistle (Carduus nutans) honey. J. Agric. Food Chem. 1993, 41, 873-878.

(8) Cotte. J.F.; Casablanca, H.; Giroud, B.; Albert, M.; Lheritier J.; GrenierLoustalof , M.F. Characterization of honey amino acid profiles using highpressure liquid chromatography to control authenticity. Anal Bioanal Chem. 2004, 378, 1342-1350.

(9) Häusler, H.; Montag, A. Minor flavour/aroma compounds in honey III. Distribution of aromatic aldehydes in floral types, and occurrence of 
heterocyclic N- and S- compounds. Dtsch. Lebensm. Rundsch. 1990, 86, $171-174$

(10) Gil, M.I.; Ferreres, F.; Ortiz, A.; Subra, E.; Tomas-Barberan, F.A. Plant phenolic metabolites and floral origin of rosemary honey. J. Agric. Food Chem. 1995, 43, 2833-2838.

(11) Ferreres, F.; Tomas-Barberan, F.A.; Soler, C.; García-Viguera, C.; Ortiz, A.; Tomas-Lorente, F. A simple extractive technique for honey flavonoid HPLC analysis. Apidologie. 1994, 25, 21-30.

(12) Cabras, P.; Angioni, A.; Tuberoso, C.; Floris, I.; Reniero, F.; Guillou, C.; Ghelli, S. Homogentisic Acid: A phenolic acid as a marker of Strawberrytree (Arbutus unedo) Honey. J. Agric. Food Chem. 1999, 47, 4064-4067.

(13) Weston R. J.; Brocklebank L.K.; Lu,Y. Identification and quantitative levels of antibacterial components of some New Zealand honeys. Food Chem. 2000, 70, 427-435.

(14) Martos, I. Caracterización Geográfico-Botánica de mieles monoflorales mediante el análisis por HPLC de sus metabolitos secundarios. 2000. Doctoral Thesis, University of Murcia.

(15) Frérot, E.; Velluz, A.; Decorzant, E.; Naef, R. From linden flower to linden honey. Part 2. Glycosidic precursors of cyclohexa-1,3-diene-1-carboxylic acids. Chem. Biodivers. 2006, 3, 94-100.

(16) Ferreres, F.; García-Viguera, C.; Tomas-Lorente, F.; Tomas-Barberan, F.A. Hesperetin, a marker of the floral origin of citrus honey. J. Sci. Food Agric. 1993, 61, 121-123. 
(17) Bonaga, G.; Giumanini, A.G.; Gliozzi, G. Chemical composition of chestnut honey: Analysis of the hydrocarbon fraction. J. Agric. Food Chem. 1986, 34, 319-326.

(18) Tomas-Barberan, F.A.; Martos, I.; Ferreres, F.; Radovic, B.; Anklam, E. HPLC flavonoids profiles as markers for the botanical origin of European unifloral honeys. J. Sci. Food Agric. 2001, 81, 485-496.

(19) Beretta, G.; Caneva, E.; Maffei Facino R. Kynurenic acid in honey from arboreal plants: MS and NMR evidence. Planta Med. 2007, 15, 1592.

(20) Mabry, T.J.; Markham, K.R.; Thomas, M.B. The Systematic Identification of Flavonoids. 1970, Springer, New York.

(21) Amirkhani, A.; Heldin, E.; Markides E.K.; Bergquist, J. Quantitation of tryptophan, kynurenine and kynurenic acid in human plasma by capillary liquid chromatography-electrospray ionization tandem mass spectrometry. J. Chromatogr. B. 2002. 780, 381-387.

(22) Beretta, G.; Caneva, E.; Regazzoni, L.; Bakhtyari, N.G.; Maffei Facino, R. A solid-phase extraction procedure coupled to ${ }^{1} \mathrm{H}$ NMR, with chemometric analysis, to seek reliable markers of the botanical origin of honey. Anal. Chim. Acta. 2008, 620, 176-182.

(23) Tomas-Barberan, F.A.; Ferreres, F.; García-Viguera, C.; Tomas-Lorente, F. Flavonoids in honey of different geographical origin. Z. Lebensm. Unters. Forsch. 1993, 196, 38-44.

This work has been funded by the European Commission; project Beeshop, FOOD CT-2006-022568. 
Table 1. ${ }^{1} \mathrm{H}$ NMR and ${ }^{13} \mathrm{C}$ NMR analysis of chestnut markers.

\begin{tabular}{|c|c|c|c|}
\hline & $\mathrm{CH} 1$ & $\mathrm{CH} 2$ & Kynurenic acida \\
\hline \multicolumn{4}{|l|}{ Protons } \\
\hline $\mathrm{H}-3$ & $6.68 \mathrm{~s}$ & $8.88 \mathrm{~s}$ & $6.63 \mathrm{~s}$ \\
\hline $\mathrm{H}-5$ & $8.09 \mathrm{brd} \mathrm{J}=\mathrm{Hz}$ & $8.28 \mathrm{brd} \mathrm{J}=\mathrm{Hz}$ & $8.08 \mathrm{~d}$ \\
\hline $\mathrm{H}-6$ & $7.32 \mathrm{brt} J=\mathrm{Hz}$ & $7.85 \mathrm{brt} J=\mathrm{Hz}$ & $7.36 \mathrm{t}$ \\
\hline $\mathrm{H}-7$ & 7.66 brt J=Hz & 7.59 brt J=Hz & $7.69 \mathrm{t}$ \\
\hline $\mathrm{H}-8$ & 7.99 brd J=Hz & 7.86 brd $\mathrm{J}=\mathrm{Hz}$ & $7.96 \mathrm{~d}$ \\
\hline \multicolumn{4}{|l|}{ Carbons } \\
\hline C-2 & 140.0 & 145.3 & 140.0 \\
\hline C-3 & 109.0 & 107.5 & 109.5 \\
\hline C-4 & 178.0 & 178.1 & 177.7 \\
\hline C-5 & 123.5 & 124.4 & 123.7 \\
\hline C-6 & 119.6 & 119.9 & 119.6 \\
\hline C-7 & 132.0 & 133.8 & 132.2 \\
\hline C-8 & 124.6 & 125.0 & 124.6 \\
\hline C-9 & 125.6 & 126.0 & 125.7 \\
\hline C-10 & 142.8 & 139.8 & 140.3 \\
\hline $\mathrm{COOH}$ & 163.8 & 166.5 & 163.6 \\
\hline
\end{tabular}

a(19) 
Table 2. Markers content in chestnut honey ${ }^{\mathrm{a}}$.

\begin{tabular}{|c|c|c|c|c|c|}
\hline Samples & $\mathrm{CH} 1$ & $\mathrm{CH} 2$ & $\mathrm{CH} 3$ & CH5 & Total \\
\hline C-00 & $40.6 \pm 0.8$ & $3.0 \pm 0.3$ & $0.1 \pm 0.00$ & $2.0 \pm 0.0$ & 45.7 \\
\hline$C-1390$ & $30.7 \pm 0.3$ & $2.4 \pm 0.0$ & $0.1 \pm 0.0$ & $0.7 \pm 0.1$ & 33.9 \\
\hline C-1454 & $35.1 \pm 0.2$ & $2.7 \pm 0.1$ & $0.2 \pm 0.0$ & $1.1 \pm 0.02$ & 39.1 \\
\hline C-1456 & $43.1 \pm 0.2$ & $3.3 \pm 0.9$ & $0.1 \pm 0.0$ & $1.0 \pm 0.0$ & 47.5 \\
\hline C-1757 & $49.6 \pm 0.5$ & $4.3 \pm 0.2$ & $0.2 \pm 0.1$ & $0.8 \pm 0.03$ & 54.8 \\
\hline
\end{tabular}

a Values are $\mathrm{mg} / 100 \mathrm{~g}$ of honey. 
Table 2. Markers content in chestnut honeya ${ }^{\mathrm{a}} \mathrm{pH}=2$

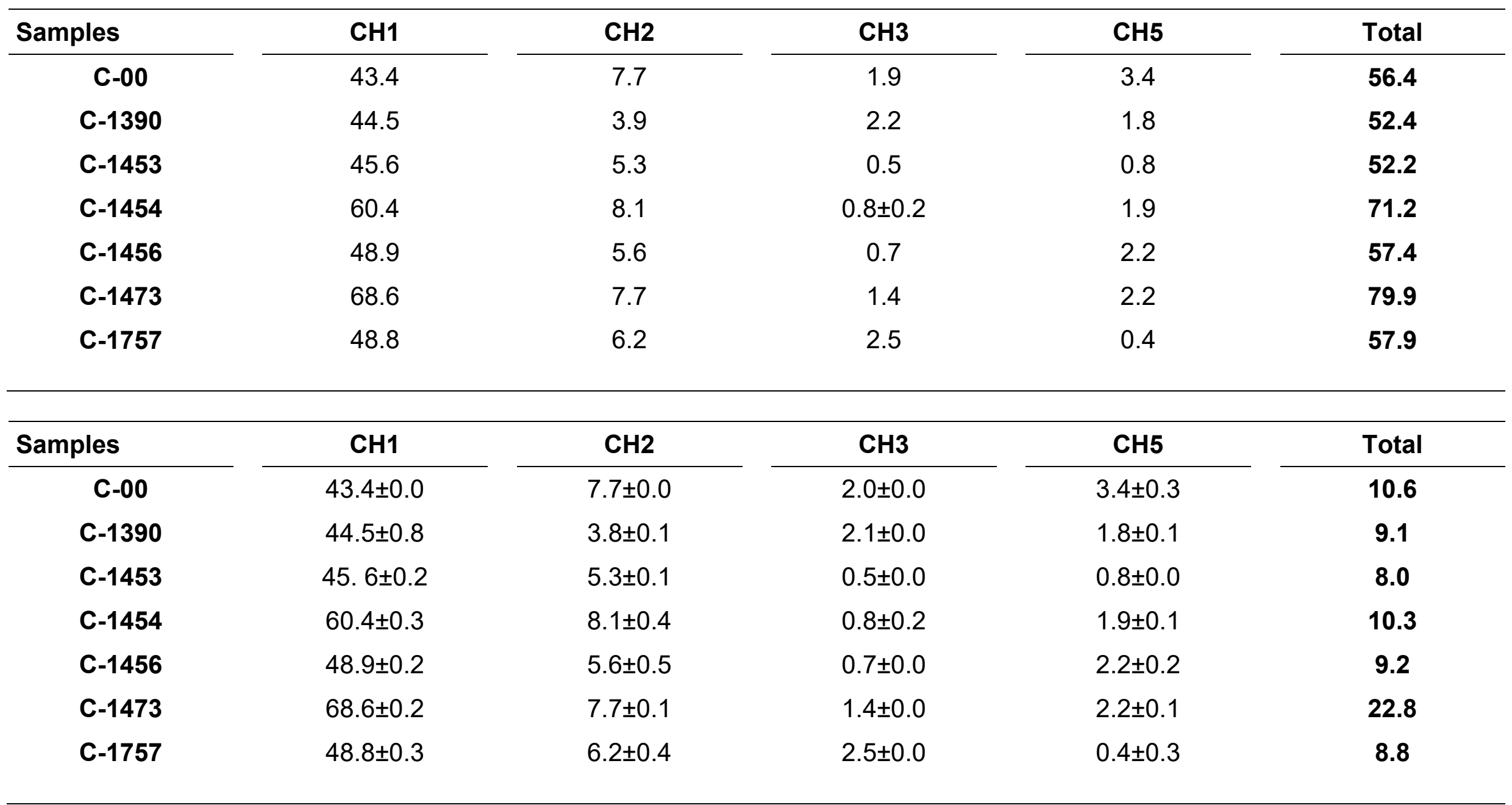


Table 2. Markers content in chestnut honeya ${ }^{\mathrm{a}} \mathrm{pH}=7$

\begin{tabular}{|c|c|c|c|c|c|}
\hline Samples & $\mathrm{CH} 1$ & $\mathrm{CH} 2$ & $\mathrm{CH} 3$ & CH5 & Total \\
\hline C-00 & 49.46 & 3.97 & & 2.23 & \\
\hline C-1390 & 44.99 & 4.06 & 0.08 & 1.00 & \\
\hline C-1454 & 60.47 & 3.81 & & 2.33 & \\
\hline C-1456 & 51.76 & 3.32 & & 1.21 & \\
\hline C-1757 & 48.43 & 3.13 & 0.07 & 1.23 & \\
\hline
\end{tabular}


Table 3. Phenolic Compounds Contents in Chestnut Honeys ${ }^{a}$.

\begin{tabular}{|c|c|c|c|c|c|c|c|}
\hline \multicolumn{8}{|c|}{ Flavonoids } \\
\hline & C-00 & C-1390 & C-1453 & C-1454 & C-1456 & C-1473 & C-1757 \\
\hline Pinobanksin & nd & $0.3 \pm 0.0$ & $0.6 \pm 0.0$ & nd & nd & nd & nd \\
\hline Methyl-quercetin & $0.1 \pm 0.0$ & $0.2 \pm 0.0$ & $0.2 \pm 0.03$ & $0.2 \pm 0.0$ & $0.1 \pm 0.0$ & $0.2 \pm 0.0$ & $0.2 \pm 0.0$ \\
\hline Chrysin & $0.1 \pm 0.0$ & $0.2 \pm 0.0$ & $0.3 \pm 0.0$ & $0.2 \pm 0.0$ & $0.1 \pm 0.0$ & $0.3 \pm 0.0$ & $0.1 \pm 0.0$ \\
\hline Galangin & $0.1 \pm 0.0$ & $0.1 \pm 0.0$ & $0.2 \pm 0.0$ & $0.1 \pm 0.0$ & $0.1 \pm 0.0$ & $0.2 \pm 0.0$ & $0.0 \pm 0.0$ \\
\hline
\end{tabular}

a Values are $\mathrm{mg} / 100 \mathrm{~g}$ of honey. nd, not detected 


\section{Figure Captions.}

Figure 1. (A) UV chromatogram of chestnut nectar at $290 \mathrm{~nm}$; (B) UV chromatogram of chestnut honey at $290 \mathrm{~nm}$ and UV spectra of the main phytochemicals. CH1, 4-hydroxyquinaldic acid (kynurenic acid); $\mathbf{C H 2}$, 4quinolone 2-carboxylic acid; $\mathbf{C H} 3$ and $\mathbf{C H 5}$, unidentified markers of chestnut honey; F, quercetin 3-O-pentosyl-hesoxide; L1, 6-O-( $\beta$-D-glucopyranosyl)- $\beta$-Dglucopyranosyl ester 4-(1-hydroxy-1-methylethyl) cyclohexa-1-3 diene-1carboxylate; L2; 4-(1-hydroxy-1-methylethyl) cyclohexa-1-3 diene-1-carboxylic acid; Pc, pinocembrin; $\mathbf{M - Q}$, methyl quercetin and $\mathbf{C h}$, chrysin.

Figure 2. HPLC-MS-MS analyses (MS') of compounds $\mathrm{CH} 1$ and $\mathrm{CH}$ 2: (A) $\mathrm{CH} 1$ in positive mode; (B) $\mathrm{CH} 1$ in negative mode; (C) $\mathrm{CH} 2$ in positive mode; (D). $\mathrm{CH} 2$ in negative mode

Figure 3. Molecular structures of compounds $\mathrm{CH} 1$ (4-hydroxyquinaldic acid), CH2 (4-hydroxyquinaldinium cation) and L1, 6-O-( $\beta$-D-glucopyranosyl)- $\beta$-Dglucopyranosyl ester 4-(1-hydroxy-1-methylethyl) cyclohexa-1-3 diene-1carboxylate.

Figure 4. $\mathrm{CH} 1$ and $\mathrm{CH} 2$ interconversion. 
Figure 1.
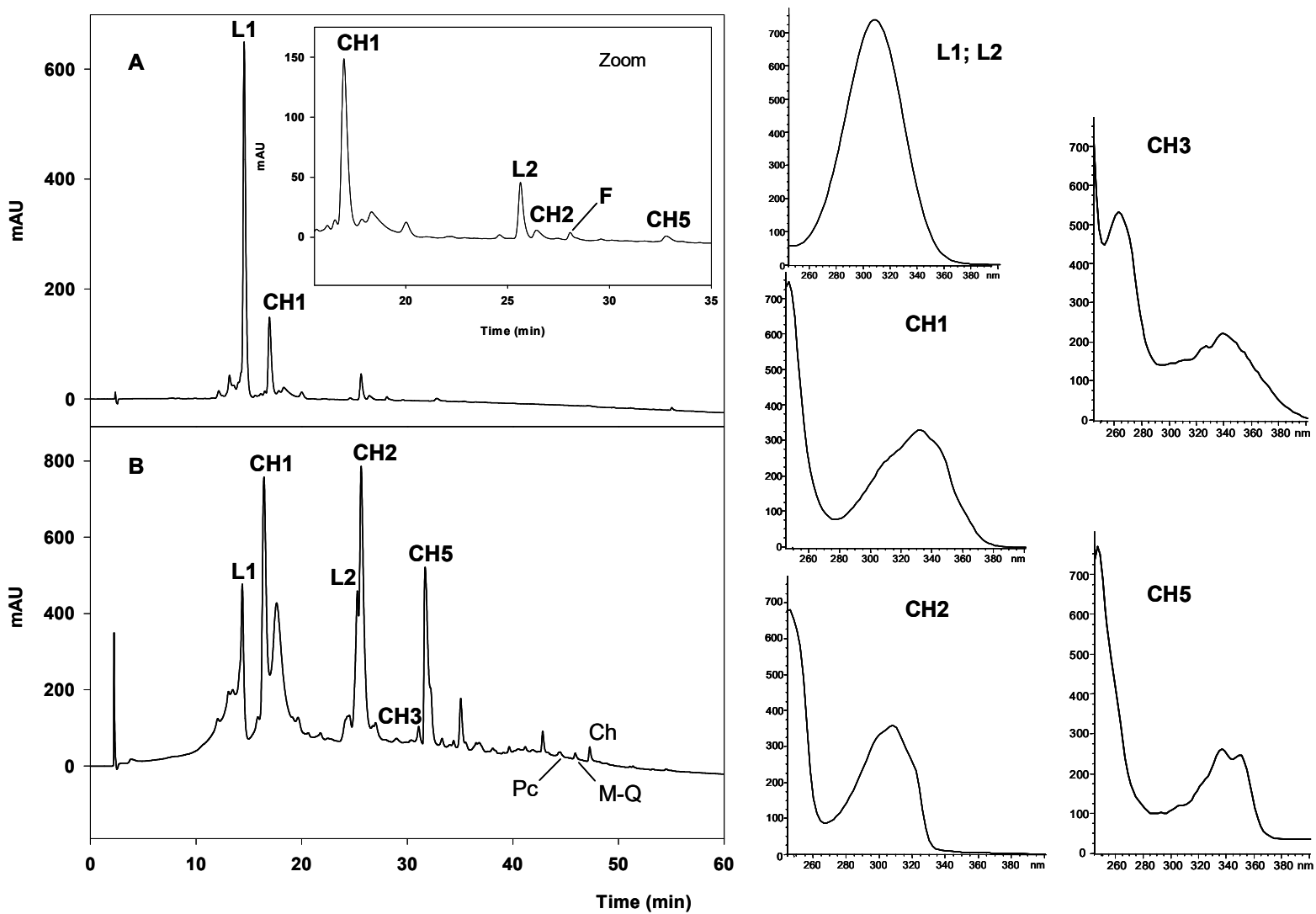
Figure 2.

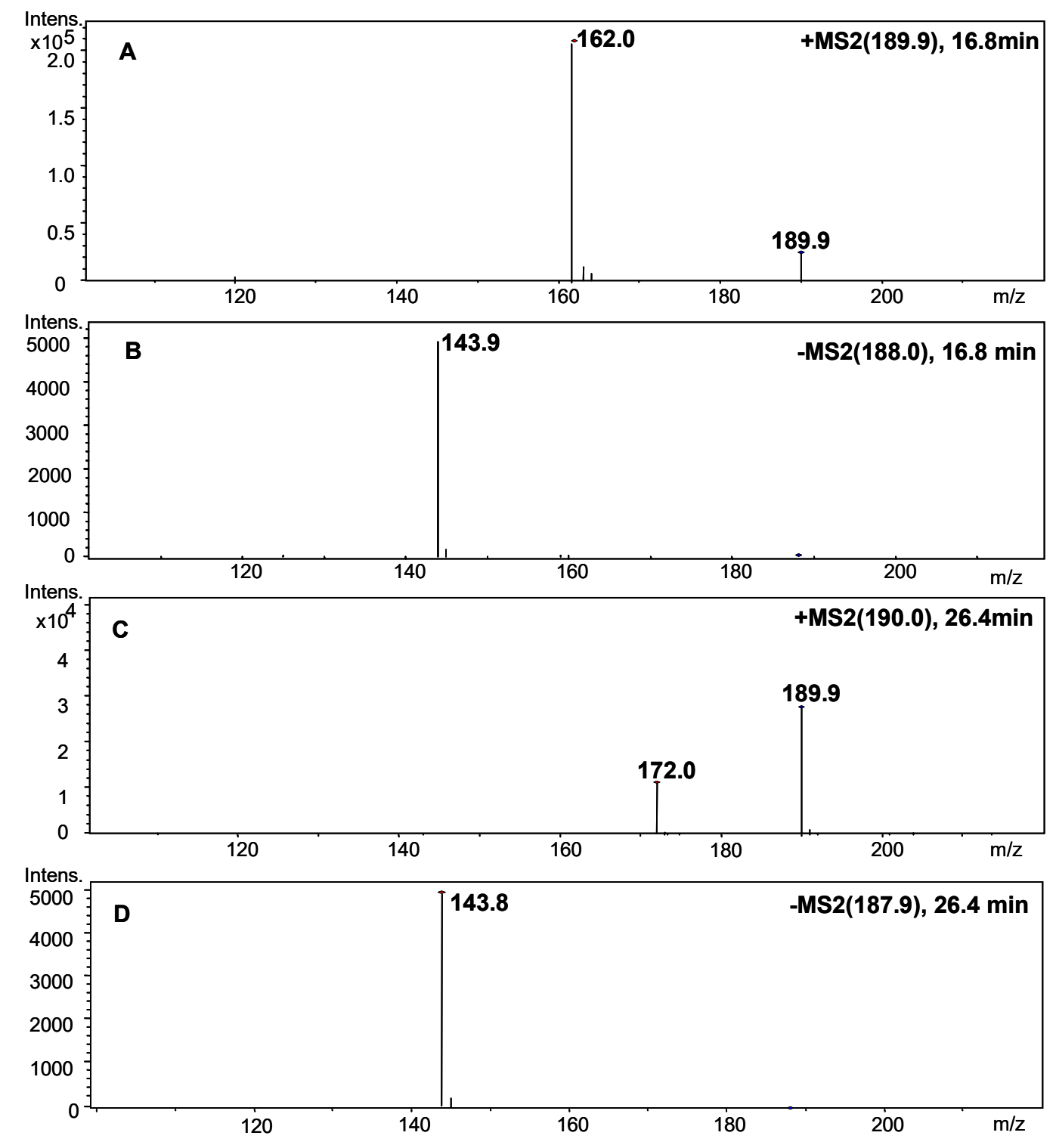


Figure 3.
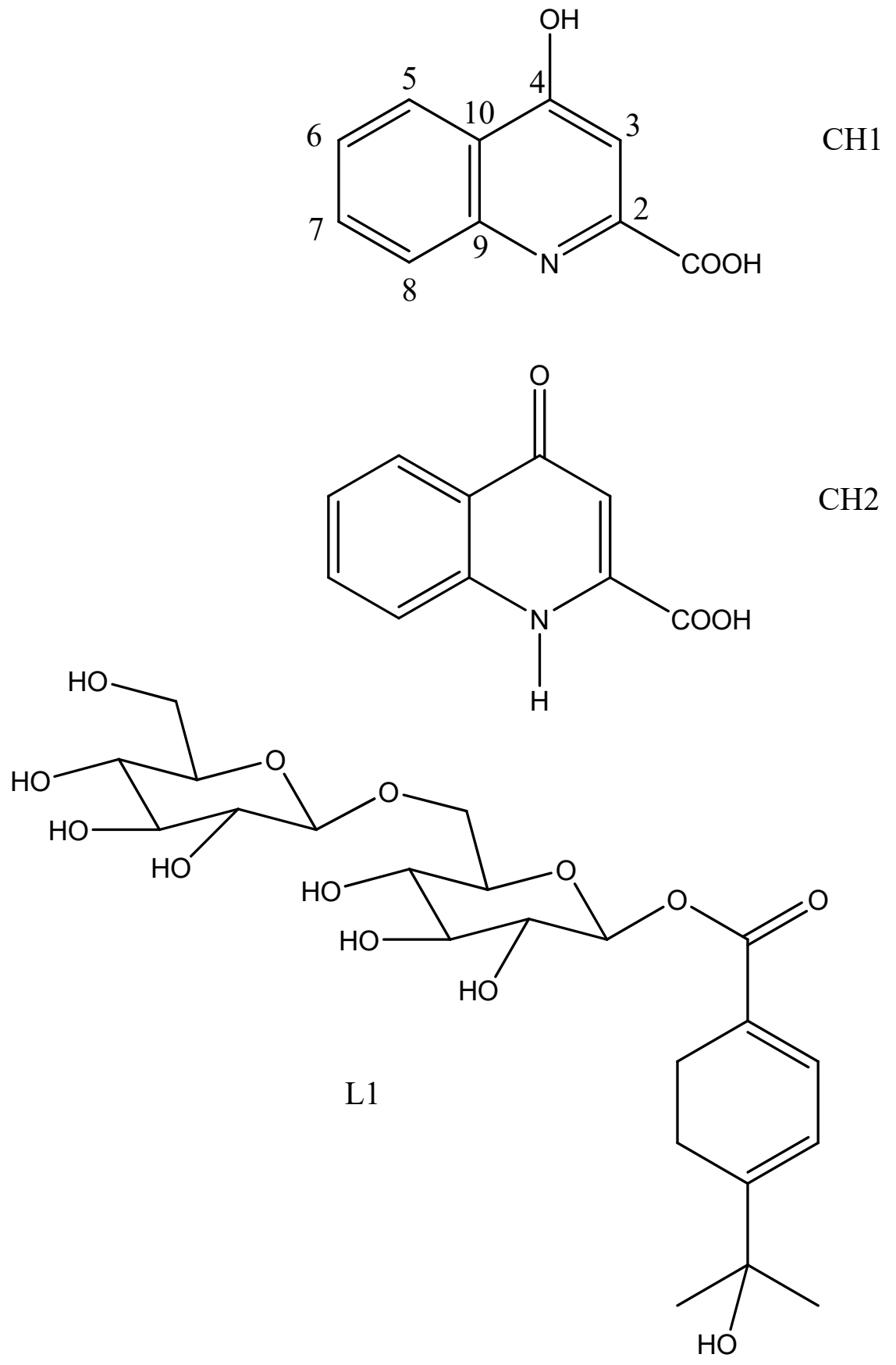